\title{
Molecular characterization of Diarrheagenic Escherichia coli strains Isolated from Dairy Calves in North Shoa Zone, Oromiya Region, Ethiopia
}

\author{
Abinet Endale \\ Addis Ababa University College of Natural Sciences \\ Yimer Muktar \\ Woldia University \\ Tesfaye Sisay Tessema ( $\nabla$ tesfu74@yahoo.com ) \\ Institute of Biotechnology, Addis Ababa University https://orcid.org/0000-0002-4103-5029
}

\section{Research}

Keywords: Calf, Diarrhea, diarheagenic Escherichia coli, pathotypes, Plasmid profile, Virulence gene

Posted Date: August 2nd, 2020

DOI: https://doi.org/10.21203/rs.3.rs-50805/v1

License: (c) (i) This work is licensed under a Creative Commons Attribution 4.0 International License.

Read Full License 


\section{Abstract}

Background Diarrheagenic E. coli (DEC) strains are associated with several outbreaks and sporadic cases worldwide. Understanding the nature of the organisms is important in order to tailor preventive and control strategies before any harm emanates. Methods In this study a total of $73 \mathrm{E}$. coli isolates from diarrheic dairy calves found in 56 farms located in North Shoa Zone, Oromiya Region, were characterized based on their virulence genes and plasmid profile. Moreover, the study showed the relationship between virulence genes, plasmid profile and antibiotic resistance traits were assessed. Results Virulence genes (VGs) were detected in $69.86 \%$ of isolates, carrying at least one of the six VGs screened. Among the VGs eae was most frequent, observed in $58.90 \%$ of isolates, followed by stx $1,34.25 \%$, stx $2,24.66 \%$, ehlyA , $23.29 \%$, bfpA, $12.33 \%$ and aat, $5.48 \%$. A total of eleven distinct virulence profiles were identified based on combination of virulence genes and isolates were placed into five different pathotypes with a frequency of EHEC, $30 \%$, tEPEC, $12 \%$, aEPEC, $16 \%$, and STEC and EAEC, $6 \%$ each. Plasmid analysis revealed the occurrence of ten different kinds of plasmids ranging in size from $2.6+0.14 \mathrm{Kbp}$ to $98.2+$ $4.17 \mathrm{Kbp}$ and exhibited 13 different kinds of profiles among the isolates. A significant one to one correlation was observed among virulence factors, plasmid content and antibiotic resistance traits with a Pearson product moment correlation in a range of $r=0.17$ to $r=0.56$. Accounting to the total dataset, isolates showed significant segregation in to the sample site, indicating spatial clustering of isolates based on overall pathogenic characteristics. Conclusions The demonstration of pathogenic potential in substantial amount of E. coli isolates from dairy farms from all the three sites indicates the level of risk posed in humans as well as animals. Thus, identifying potential sources and route of transmission of DEC is vitally important in order to establish control and prevention strategies for diarheagenic E. coli infection.

\section{Introduction}

Escherichia coli used to be regarded as a harmless commensal to mammals ever since its first discovery in 1885 by Theodor Escherichia [13]. However, consecutive outbreaks in the developed world placed the organism under close scrutiny among public health authorities and researchers with the identification of extremely diverse pathogenic varieties that cause diseases in humans and animals $[31,13]$.

Currently pathogenic strains of $E$. coli are known to cause a wide variety of diseases in wide range of hosts, infecting sites such as; the urinary tract, blood stream, central nervous system, in addition to the commonly inflicted gastro intestinal tract (GIT) $[31,13]$. Pathogenic $E$. coli strains are now considered to be a major public health threat due to their extraordinary transmission potential through survival in diverse habitats; thus, food, water, animals and contaminated environment are implicated as potential sources for the transmission of the organism [5].

More than six pathotypes of intestinal pathogenic E. coli or diarrheagenic E. coli (DEC), distinguished by possessing diverse virulence factors (VFs) encoded by specific virulence genes (VGs), were recognized so far. These include: Enteropathogenic E. coli (EPEC), Shigatoxigenic E. coli (STEC), Enterotoxigenic E. coli 
(ETEC), Enteroaggregative E. coli (EAEC), Enteroinvasive E. coli (EIEC) and Diffusely Adherent E. coli (DAEC) $[4,33,16]$. These virulence factors confer colonization, fitness and overall pathogenic potential to the organism [23]. Even though all pathotypes contribute to diarrhea; mechanism of colonization, symptoms and clinical outcomes vary considerably, exemplifying the diversity of the organism [12]. The different pathotypes of DEC, were thoroughly reviewed in the comprehensive reviews by Nataro and Kaper [31] and Croxen et al. [13].

In general, diarrhea causes considerable morbidity and mortality worldwide, particularly in children under the age of five [26] and calves [25]. While there are many etiological agents responsible for diarrhea, DEC takes the major share as it is the most frequently isolated organism in diarrheal patients [27]; it is also the most common cause of diarrhea and septicemia in calves [25]. Although DEC were found to be the most common cause of bacterial diarrhea in many parts of the world, still to date comprehensive picture of DEC incidence as well as bacterial and host factors that predispose to disease are largely unknown especially in the developing world $[9,26]$. However, the disease burden is expected to be high in Africa, given that food-borne disease occurs commonly in developing countries because of inadequate food safety laws, weak regulatory system, poor food handling and sanitation practices [26, 34].

Previous efforts by the scientific as well as clinical communities lead to great successes in understanding the biology, ecology and pathology of these organisms. These advances helped clinicians for quick and appropriate management of associated illness. Nonetheless, the high prevalence rate and the worrisome emergence of antimicrobial resistance and high asymptomatic carriage rates in diverse hosts and reservoirs, makes the clinical management difficult $[8,11,18]$.

Like the rest of Africa, surveillance for various types of DEC is overlooked in Ethiopia. So far only limited studies were done on the prevalence and burden of associated illnesses in Ethiopia, indicating the need for local studies for understanding the distribution of various DEC types and associated health impact. Therefore, this study was carried out to determine diarrheagenic $E$. coli strains and their plasmid profiles from diarrheic calves in Muketuri, Debretsige and Fiche towns of North Shoa Zone.

\section{Results}

\section{Virulence genes present in diarheagenic $E$. coli}

Virulence gene specific bands (Fig. 1) were totaled for each isolate under each category. Overall results showed 51 isolates, about $70 \%$ of the total 73 E. coli samples, were found to carry at least one of the targeted virulence genes. This figure is significantly high $(p=0.001$, One-Sample Binomial test compared to 22 isolates (30\%) that carried no VGs, which indicate high level of pathogenic potential in the sample population.

Results revealed that eaeA, which codes for intimin in both EHEC and EPEC was found to be the single most frequent VG, detected in $59 \%$ of the isolates (Table 2). While the st 1 primarily carried by STEC and also by EHEC was the second most common VG, detected in $25(34 \%)$ of isolates followed by stx 2 and 
ehly $A$ which were detected in 25 and 23 percent of the total sample isolates respectively. Whereas, EPEC bundle-forming pili gene $(b f p)$ and the EAEC transcriptional regulator gene (aatA) were detected in $12 \%$ and $5 \%$ of isolates, respectively (Table 2 ).

Furthermore, occurrence of $E$. coli virulence genes was described in terms of spatial distribution. It was found that, Muketuri site has the lowest overall frequency of VGs. In contrast, Debretsige has the highest occurrence of VGs. Among the VGs screened in this study, eaeA was most frequently detected across all the sites, occurring in Debretsige in $86 \%$, Fiche in $64 \%$ and Muketuri in $46 \%$ of the sample population. On the other hand, stx 1 gene was the second most commonly detected genes across all sites after the eaeA. Among the three sites, Debretsige has the highest occurrence of bfpA (43\%) compared to the other two sites which had occurrence of the gene in around $5 \%$ of isolates. Among the VGs aatA, which is the list frequent, was detected only in the two of the sample sites (Muketuri and Fiche) detected in $8 \%$ and $5 \%$ of isolates, respectively. Comparative analysis of the distribution of VGs across all the three sites is presented in Fig 2. below.

The average number of virulence genes per sample isolate was found to be $1 \pm 0.34$ (media \pm standard deviation). Out of the total isolates carrying virulence genes, $16(31 \%)$ of the isolates were found to carry two VGs, while $39 \%$ of isolates carried 3 or more VGs. Among the isolates that had a single virulence factor, eae $A$ was the most frequent which is observed in 12 of the 15 isolates. Two of the remaining isolates had either of aatA or Stx 1 as a single VG. The remaining VGs were detected in combination with other VGs, out of which 31 (61\%) had eaeA in combination with one of the other VGs. While the other combinations were Stx 1 with Stx23 isolates (6\%) representing typical STEC and two isolates (4\%) showing peculiar combination of Stx1 with aatA (Table 2).

Based on the difference in combination of the six virulence genes, eleven virulence profiles (VPs) have been identified (Table 3). Isolates carrying a single VG, any one of eaeA, Stx 1 or aatA were categorized in to three different VPs (VP-I, VP-II and VP-III). While isolates carrying two VGs showed 4 different VPs carrying two of the six virulence factors in different combination. Similarly, three VPs were identified in isolates carrying $3 \mathrm{VGs}$.

On the basis of combinations of VGs, the $E$. coli isolates were further placed into one of four main pathotypes (STEC, EPEC, EHEC and EAEC) and two subtypes of EPEC, tEPEC and aEPEC. Whereas isolates that were found negative for the presence of VGs investigated in this study were designated as un-identified (UI), hence the markers used in the study were not exclusive to detect all previously identify pathotypes of DEC.

Accordingly, approximately $70 \%$ of isolates were classified as distinct pathotype and the remaining $30 \%$ were not placed under defined pathotype, thus designated as un-typeable (UI). Isolates belonging to the EHEC subtype were most commonly detected representing 22 isolates (30\%), followed by EPEC (28\%) where, aEPEC and tEPEC subtypes constituting $16 \%$ and $12 \%$ respectively. Whereas, STEC and EAEC showed equal prevalence of $6 \%$ represented by 4 isolates each. The percentage distribution of $E$. coli pathotypes are shown in Fig 3. 
Furthermore, the prevalence of E. coli pathotypes was described in terms of spatial distribution. The distribution of $E$. coli isolates with defined pathotypes from the three sampling locations is shown in Fig 4 below. From the isolates that were designated as distinct pathotype, EHEC was most frequently detected in Fiche and Debretsige represented by $45 \%$ and $36 \%$ of samples isolates collected from the sites respectively. Similarly, Debretsige has the highest prevalence of tEPEC representing $43 \%$ of sample isolates. Whereas STEC was detected only in Muketuri site and EAEC has not been detected in Debretsige.

\section{Plasmid Profile of diarrheagenic E. coli isolates}

In order to further characterize the DEC isolates, 30 samples were randomly selected from isolates that were found to carry at least one of VGs and investigated for their plasmid content. Plasmid DNA were extracted from sample isolates and classified according to their size by electrophoresis on $0.7 \%$ agarose gel alongside $1 \mathrm{Kbp}$ DNA marker. Analysis of isolate's plasmid content revealed the presence of ten different kinds of plasmids, with sizes ranging from $2.5 \pm 0.13 \mathrm{Kbp}$ up to around $98.2 \pm 4.17 \mathrm{Kbp}$ as measured by an open access software GelQuant ${ }^{\circledR}$ (Fig 5).

The number of plasmids per sample isolate varied from 1 to 5 and all of the isolates were found to carry at least one plasmid, were the largest plasmid $98.2 \pm 4.17 \mathrm{Kbp}$ in size were observed in all isolates that were screened for their plasmid content. Whereas, the smaller plasmids showed inconsistent distribution among sample isolates; therefore, regarded as putative plasmids. Among the putative plasmids, plasmid size of $16 \pm 1.4 \mathrm{Kbp}$ was most frequently observed, detected in $14(47 \%)$ isolates. While plasmid size of $35 \pm 1.7 \mathrm{Kbp}$ was the least frequently detected, occurring in 2 of the isolates $(7 \%)$. The distribution of plasmids and their frequency is summarized in table 4 below.

Plasmid distribution show considerable variation among the three sample sites (Table 5). Among the identified plasmids, plasmid size $11.8 \mathrm{Kbp}$ and $34.6 \mathrm{Kbp}$ were detected only in Fiche, while plasmid size $2.5 \mathrm{Kbp}, 14.3 \mathrm{Kbp}$ and $42.5 \mathrm{Kbp}$ were not observed in this locality. Similarly, plasmid size around $5 \mathrm{Kbp}$ was unique for Muketuri and Fiche, while the remaining plasmids were detected in common among the three sample sites.

Based on the difference in the distribution of identified plasmids, twelve distinct plasmid profiles (PPs) have been identified. The most commonly occurring plasmid profile was plasmid profile-I observed in 13 $(43.33 \%)$ isolates followed by plasmid profile IV and VIII each occurring in $3(10 \%)$ isolates (Table 6$)$.

\section{Association Between Plasmids, Antimicrobial Resistance and Virulence Factors.}

In previous work on the isolates, the samples used in this study were tested for antibiotic resistance against eleven antibiotics and all isolates showed resistance against one or more antibiotics (Under 
preparation). Based on combination of antibiotic resistance traits, the sample isolates exhibited 27 different resistance profiles (RT-1 to RT-27) (Data not provided). These data were used together with the data generated in this study to investigate any possible association between plasmid content, antimicrobial resistance and virulence factors in test isolates.

Correlation between antimicrobial resistance and plasmid distribution revealed a positive correlation $(r=$ $0.32 ; p<0.01$ ) between the two variables (Fig 6.a). These can be observed by significantly high frequency of plasmids in resistant strains $(p<0.05)$ than susceptible strains. It is also found that, isolates resistant to multiple antibiotics ( 4 or more drugs) were found to carry 3 or more plasmids on average. Chi-squared distribution was also employed to identify the level of significance between a plasmid profile and antibiotic resistance trait. In this regard, several plasmid profile types occurred in a significantly higher proportion of resistant isolates than their susceptible counterparts. The analysis identified plasmid profiles associated with the greatest numbers of resistance traits, include PP-II (resistance to $E, S$, K, and AMP), PP-VIII (resistance to E, S, and AMX) and PP-IX (resistance to E, S, FOX and TET). However, multivariable logistic regression applied to identify any significant associations between a particular plasmid type and resistance trait, showed no significant association, as no particular plasmid can be directly associated to specific drug resistance trait.

Moreover, strong correlation was observed between virulence factor content and both plasmid profile and drug resistance trait. Results show the number of plasmids in an isolate were positively correlated with number of virulence gene content $(r=0.56 ; p \leq 0.044)$. This is depicted in Fig 6.b. Similarly, virulence gene content of an isolate was positively correlated with antibiotic resistance trait, even though it is found to be statistically insignificant. This can be explained by a $20 \%$ higher level of antibiotic resistance observed among isolates carrying one or more virulence gene compared to isolates that doesn't have any VG or $60 \%$ increase in antibiotic resistance from isolates with single VG to isolates carrying 4 VGs (Fig 6.c).

The values for each pairwise combinations of the first three axes plotted as a series of Cartesian grids show the relationship among the three variables in two-dimensional space (Fig 7).

Multivariate analysis of variance (MANOVA) conducted to determine whether the groups differed significantly according to the first three PCoA axes and significant difference was observed. In order to identify the factor responsible to the difference, univariate ANOVA was used by grouping the three arises against sample site and sample source variables such as age, sex and breed type of calf. The pairwise comparisons of individual groups according to each PCoA axis revealed significant difference $(p<0.05)$ only across sample sites.

\section{Discussion}

The overall occurrence of virulence genes observed in this study is significantly high compared to 22 isolates $(30 \%)$ that carried no virulence genes. The result is comparable with what is reported by [43], occurrence of virulence genes in $76.45 \%$ of fecal samples collected from 824 diarrheic calves in Iran. But it is comparatively high compared to $30.1 \%$ occurrence of isolates carrying at least one of the eight 
virulence genes from 620 calves investigated by Piccoa et al. [38] in Cordoba province, Argentina and $15.2 \%$ prevalence of virulence genes reported in Australia [29]. This indicates high level of virulence factor gene carrying $E$. coli in the sample area, indicating high level of pathogenic potential in the sample population.

The high frequency of virulence genes observed in the current study is mainly attributed to high occurrence of eaeA (59\%), that cods for an adhesin protein intimin in both EPEC and EHEC. This result is in agreement with previously reported occurrence of this gene in $38 \%$ of diarrheic calve fecal samples in Iran by Shahrani et al. [43] and 57.1\% from Australian calves by Luna et al. [29]. But it is by far more than $6 \%$ prevalence of eae in Mediterranean water buffalo calves in Italy Borriello [6]. Intimin is a $94-\mathrm{kDa}$ protein encoded by the eae gene on locus of enterocyte effacement and it is found in all strains capable of inducing $\mathrm{A} / \mathrm{E}$ histopathology [23].

The locus of enterocyte effacement also harbors additional virulence factors such as T3SS which provide the pathogen leverage over the normal intestinal flora in the competition for intestinal site by intimate adhesion and biofilm formation, favoring overgrowth of pathogen at low infection dose $[15,40]$. Thus, the relative abundance of A/E E. coli; EHEC (28\%) and EPEC (30\%) in isolates of the current study can be explained by the presence of LEE in this isolates and combined effect of different virulence factors encoded on it.

In the current study comparably, higher frequency of stx 1 harboring STEC strain were observed in sample area. As shiga like toxin I is reported to be more often detected than shiga like toxin II in STEC strains, the relative abundance st 1 in current investigation is in agreement with most reports of studies conducted around the world. For instance, Seyda et al. [41], showed that all STEC isolates from cow with mastitis harbor stx 1 gene and identified stx 2 only in $20 \%$ of the STEC isolates. Similarly, high frequency of $E$. coli isolates carrying the stx 1 was observed in $86.67 \%, 40.7 \%, 12.2 \%$ E. coli isolates from diarrheic calves compared to $26.67 \%, 28.3 \%, 7.8 \%$ of $s t \times 2$ in Egypt, Iran and Australia respectively [19, 43, 29]. In contrast higher prevalence of $33 \%$ stx 2 gene compared to $17 \%$ stx 1 gene in cattle has been reported in Iran [1].

Epidemiological studies revealed that $s t \times 2$ is more associated with severe human disease such as; $\mathrm{HC}$ and HUS than st 1 [13]. In one study for instance, st $\times 2$ and a variant, st $\times 2 \mathrm{c}$, were the only subtypes found from HUS cases [17]. Thus, even though $s t \times 2$ is less frequently detected than $s t \times 1$, the overall occurrence of $s t \times 2$ among sample isolates is $25 \%$, indicating a potential risk for severe illness following DEC infection in the areas.

In addition to Shiga-like toxin, virulence potential of STEC strains is also determined by additional virulence factors. Among others, virulence factors encoded on LEE had been well characterized so far and it is associated with to A/E lesion in the host resulting sever illnesses such as HUS and HC $[39,15]$. In this regard considerable amount of STEC strains 22 of the 28 STEC isolates $(78.57 \%)$ were found to contain eae and additional virulence factors that are harbored in locus of enterocyte effacement, a pathogenic island that harbored the gene, indicating the associated potential risk following DEC infection in the area. 
This result is in agreement with results obtained by Seyda et al. [41] who identified eae in $40 \%$ of STEC isolates.

LEE harboring STEC strains stem as distinct subtype EHEC Nataro and Kaper [31], 22 of the isolates in the current investigation can be regarded as EHEC. Among the EHEC isolates $18(50 \%)$ harbored only stx 1 and $2(5.6 \%)$ isolates carried stx 2 as a single Stx gene. While the remaining 16 isolates $(44.4 \%)$ contained both stx1/stx2 Virulence genes. This result is inconsistent with the findings of Badouei et al. [1] who identified $15.4 \%$ isolates to harbored $s t x 1$ and $56.4 \%$ carried $s t x 2$ as a single Stx gene. While the remaining contain both stx 1 and stx 2 from 39 EHEC isolates collected from cattle in Iran. Similarly, Borriello [6] identified STEC isolates from fecal samples of water buffalo calves, were all Stx and intiminpositive, with Stx1 (80\%) more frequent than Stx2 (27\%).

In Addition to shiga-like toxins, EHEC strains may carry the EHEC hemolysin toxin encoded by hlyA or ehx. In the current investigation ehlyA was detected in 23 percent of the total isolates. In similar study, Luna et al. [29] identified Ehly genes in $56 \%$ of isolates from diarrheic calves in Australia. This is a pore-forming toxin that lyses erythrocytes and found to be cytotoxic to endothelial cells contributing to the development of $\mathrm{HC}$ and HUS [1] indicating a great potential risk posed by these isolates.

The other virulence gene, EAEC transcriptional regulator gene (aatA) were detected in $5.5 \%$ of isolates. In contrast the previously mentioned study in Australia, showed the occurrence of this VG in $29 \%$ of isolates [29]. This result would be an evidence for the presence the said pathotype in the study area.

On the basis of combinations of Virulence genes, the $E$. coli isolates characterized in this study were further placed into one of the three pathotypes of DEC; STEC, EPEC, EAEC and two subtypes of STEC and EPEC each.

Approximately $70 \%$ of $E$. coli investigated in this study were classified as distinct pathotype based on combinations of virulence genes detected. The recognized pathotypes, $6 \%$ STEC, $30 \%$ EHEC, $12 \%$ tEPEC, $16 \%$ aEPEC, $6 \%$ EAEC were found to be comparable with similar studies such as; $9 \%$ STEC, $26 \%$ EHEC, $12 \%$ EPEC in Iran [43] and 2.2\% tEPEC, 6.7\% aEPEC, 48.9\% STEC, $6.7 \%$ EAEC and $2.2 \%$ of both ETEC and EIEC in Egypt [42]. Similarly, studies made in Egypt that tried to characterize diarrheagenic E. coli virulence genes in newborn calves, identified different set of virulence genes; ST; $33.3 \%$, LT; $30 \%$, Stx1; 86.67\%, Stx2; $26.67 \%$ and two ETEC adhesions (F5; 13.3\%) and (F41; 16.67\%) [19].

The overall occurrence of the different virulence factors in $E$. coli isolates of the current investigation show the potential risk posed by these organisms not only to humans and domestic animal but also for wild animals. The potential transmission among different sources has been demonstrated by Miko et al. [30] who isolated 140 STEC strains from game animals in Germany between 1998 and 2006 and compared with 101 STEC isolates from farm animals, their feed products and human patients. The result showed genes linked to high-level virulence for humans (st×2, st×2, and eae) belong to the same genotypes and other virulence attributes, regardless of origin. 
Moreover, the current study tried to investigate plasmid content of study isolates as plasmids within DEC have been shown to carry both antibiotic resistance genes as well as virulence factor genes among others [14]. The study has identified ten different kinds of plasmids, with size ranging from $2.6 \pm 0.14 \mathrm{Kbp}$ to $98.2 \pm 4.17 \mathrm{Kbp}$. In agreement with the current study, Nsofor and Iroegbu [32] isolated E. coli plasmids from domestic animals, that harbor one or more plasmids with molecular size in the range of 1 to 120 Kbp. Similarly, 7 different plasmids $2.4 \mathrm{kbp}$ to $65 \mathrm{kbp}$ in size were isolated from 1,266 fecal specimens derived from environmental, human, animal and food sources [48].

Among the various plasmids identified, a large plasmid with slight variation in size has been observed in all isolates examined for their plasmid content. DECs are known to harbor large conserved plasmids associated with virulence potential as well as resistance trait $[28,7]$. For instance, the presence of large conserved plasmid is established in DEC pathotypes EAEC, EHEC and EPEC which are pAA, p0157 and pEAF respectively [13]. The size variation in the large conserved plasmids is also likely. For instance, the large plasmids of STEC were shown to vary between $115 \mathrm{Kbp}$ and $65 \mathrm{Kbp}$ in different studies $[47,35,48]$.

The smaller plasmids identified in the current investigation were regarded as putative due to their inconsistent distribution, even among isolates with similar characteristics in terms of virulence factors as well as antibiotic resistance profile. However, the study identified high level of antibiotic resistance in isolates containing these plasmids. Though results cannot be directly compared, as different methods employed, Bello et al. [2] (2013) and Kalantar et al. [22] detected plasmids more frequently in resistant strains than susceptible strains. Bello et al. [2] for instance isolated a total of 48 different plasmids occurring in various combinations from $50 \mathrm{E}$. coli isolates that showed MDR. Similarly, resistance genes for MDR were reported in E. coli plasmids by Kalantar et al. [22].

Even though plasmids were found to be strongly correlated with resistance phenotype, no particular plasmid was found to be significantly associated with a particular drug resistance phenotype. This result confirmed that there was no a linear correlation between the plasmids content and the antibiogram studies of the isolates, which suggests resistance genes might be located in various plasmids.

Antibiotic resistance is not only conferred by plasmids, studies also show inherent resistance to antibiotics by chromosomally encoded resistance genes [46]. Moreover, the large conserved plasmid of STEC strains has been shown to harbor genes resistance for antibiotics [14]. These facts potentially explain the observed antibiotic resistance despite the absence of putative plasmids in PP-I isolates.

Studies made in understanding the immense antibiotic resistance in $E$. coli indicated plasticity of $E$. coli genome and the role of HGT by mobile genetic elements such as plasmids in conferring MDR and the possibility for the transmission of these plasmids among distantly related microbial population [46]. Thus, the results of the current study indicate the need for early intervention to prevent further spread of DEC in the sample areas in order to, as the occurrence of such high level of mobile genetic elements in highly resistant bacteria presents a serious challenge in transmission of antibiotic resistance among other pathogens. 
Similarly, the significant positive correlation between antimicrobial resistance and virulence gene content showed in this study agrees with what is reported by Zhang et al. [49] who showed the prevalence of resistance to single and multiple antibiotics to be significantly higher for pathogenic isolates than for commensal isolates. In contrast, Seyda et al. [41], identified no significant differences in virulence genes between antibiotic-resistant and antibiotic-susceptible strains. However, the observed significant correlation between virulence and antibiotic resistance in this study can be explained by the effects of selection pressure and genetic association between these factors according to Zhang et al. [49] and Schroeder et. al. [40]. This study can also be taken as additional evidence for the positive correlation between antibiotic resistance and virulence factor carriage similar to the results obtained by Zhang et al. [49].

Furthermore, this study showed significant correlation between plasmid carriage and virulence factor, even though all the virulence genes used in the study are not plasmid encoded, where $80 \%$ of the putative plasmids were detected in intimin containing isolates. This finding may explain the abundance of EHEC among test samples, as extra virulence factors like additional adhesins can be conferred by plasmid encoded Virulence genes. DEC plasmids are known to be associated with virulence potential [28, 7]. Such strong association between plasmids and virulence factors may be explained based on pathogenomics and concurrent evolution according to Johnson and Nolan [20].

Based on all the study variables i.e. each of virulence factors, plasmids and antibiotic resistance traits, clear clustering of isolates across sample sites was observed indicating significant phenotypic difference across sampling sites. Such clustering of isolates in spatial and seasonal difference has also been reported on the incidence of E. coli strains in diarrheic calves by Shahrani et al. [43] in Egypt and Sidhu et al. [44] in Australia. This clustering of isolates among sites may be explained by similar sources of infection in the area as a result of environmental contamination by contaminated litters and asymptomatic carriers or as a result of difference in geography, climate and antibiotic consumption trend among sample sites.

Despite the valuable information generated in this study, the study had limitations in showing all the relevant information to get the clear picture of the different DEC pathotypes, their biology and distribution as a single host can be infected by multiple pathotypes of DEC. This is mainly the result of limited resources available to conduct the research.

\section{Conclusion}

Despite the inherent limitations, this work provides valuable information on the occurrences of major DEC virulence factors in significantly high level in diarrheic calves in the study area. Furthermore, the study demonstrated significant association between antibiotic resistance, plasmid content and virulence gene content of DEC indicating potential risk of associated infections in the areas. The role of calves as a reservoir and their feces as a source of contamination for food, environment and humans suggests, these hosts have high potential as for transmission of antibiotic resistance in addition to be source of infection. 


\section{Materials And Methods \\ Study Design}

Experimental study was conducted to characterize $E$. coli isolates based on their virulence factors and plasmid contents. The isolates were obtained from Dr. Tesfaye Sisay (Supervisor of this work, Institute of Biotechnology, AAU). Samples were collected from diarrheic calves that were exhibiting signs of systemic disease and clinically identified as diarrheic at the time of sampling. The calves were located in 56 dairy farms, composed of three large and 53 smallholder dairy farms found in Muketuri, Debretsige and Fiche towns of North Shewa Zone, Ethiopia. These towns are located in North-West of Addis Ababa. Fitche town is $147 \mathrm{~km}$ away from Addis Ababa, while Muketuri and Debretsige are $78 \mathrm{~km}$ and $89 \mathrm{~km}$ North-West of Addis Ababa, respectively. In 2010 there have been 3.3 million cattle in North Shewa Zone; where cattle account for the larger proportion (53\%) of livestock in the area. These areas are among the major suppliers of milk to the capital, where 13000 liters of milk is collected from Debretsige town only.

The samples for this study were 84 isolates, previously collected and identified as $E$. coli and stored in glycerol stocks at $-20^{\circ} \mathrm{C}$. These samples originated from $27(36.98 \%)$ cross breed calves and $46(63.01 \%)$ exotic breed calves. The age distribution of calves ranged from 1-16 weeks; where, 40 (54.79\%) were 1 - 4 weeks old; 29 (39.73\%) were 4 - 8 weeks old and 4 (5.48\%) were more than 8 weeks of age. The calves were $26.00 \%$ male and $74.00 \%$ female; calves were sold soon after birth and females were kept for replacement stock in all dairy farms.

\section{Bacteria}

Glycerol stocks of $E$. coli isolates were thawed and sub-cultured overnight at $37^{\circ} \mathrm{C}$ on MacConkey agar (Himedia, India) and further confirmed for purity by sub-culturing on Eosine methylene blue (EMB) agar (Himedia, India) as previously described in O'Sullivan et al. [33]. All viable samples at the time of the experiment ( $n=73$ ) were screened for virulence genes (VGs). All of the samples were previously tested for their susceptibility against a panel of eleven antibiotics. Accordingly, all of the isolates were found to be resistant to at least one of the antibiotics tested and 27 different types of resistance profiles were obtained (under preparation for publication).

\section{Virulence Gene Detection}

Genomic DNA was extracted from single colony of bacterial isolates grown on EMB medium that were then transferred into nutrient broth cultured at $37^{\circ} \mathrm{C}$ overnight. Culture $(1.5 \mathrm{ml})$ was spun by centrifugation at $5000 \mathrm{~g}$ for $10 \mathrm{~min}$ and the bacterial pellet was mixed with $50 \mu \mathrm{l}$ of sterile distilled water and lysed by boiling in a water bath at $95^{\circ} \mathrm{C}$. The lysate was centrifuged again as mentioned earlier. The supernatant was then directly used as a template for PCR. 
For all isolates, PCR based screening of DEC VGs were performed as previously described by Pal et al. [36] and Khan et al. [24] with minor modifications. Briefly, PCR reaction mix of $25 \mu \mathrm{l}$ was prepared by mixing $2.5 \mathrm{ml}$ of tenfold reaction buffer (Himedia, India), $1 \mu \mathrm{l} 0.25 \mathrm{mM}$ of each of the four dNTPs, 0-1.5 $\mathrm{mM} \mathrm{MgCl} 2,1 \mathrm{U}$ of Taq polymerase enzyme (Himedia, India), $3 \mu \mathrm{l}$ of $100-400 \mathrm{ng} / \mathrm{ml}$ extracted DNA template and $0.5 \mu \mathrm{mol} \mathrm{m} 1^{-1}$ of respective primers (Table 1 ), and filled with ultrapure water. Ultrapure water, instead of template DNA, was used as a negative control.

The PCR reaction mixtures were incubated in a thermo cycler programmed with the thermal profile described by Paton and Paton [37] with slight modification. Briefly initial denaturation at $95{ }^{\circ} \mathrm{C}$ for three minutes was followed by 35 cycles of each consisting 1 min of denaturation, annealing and extension. Denaturation and extension temperatures were $95^{\circ} \mathrm{C}$ and $72{ }^{\circ} \mathrm{C}$, respectively. While the annealing temperatures were $55^{\circ} \mathrm{C}, 45^{\circ} \mathrm{C}, 55^{\circ} \mathrm{C}, 54^{\circ} \mathrm{C}, 46^{\circ} \mathrm{C}, 48^{\circ} \mathrm{C}$ for stx 1 , stx2, eaeA, bfpA, ehlyA and aat $A$, respectively. Following the $35 \mathrm{PCR}$ cycles, each sample was subjected to final extension at $72{ }^{\circ} \mathrm{C}$ for 10 $\min$.

Amplified PCR products were separated by gel electrophoresis at 10 volts per $\mathrm{cm}$ for 45 minutes in $1 \mathrm{x}$ TBE buffer, in $1 \%$ agarose gel (Himedia, India) containing $0.5 \mathrm{~g} \mathrm{ml}^{-1}$ ethidium bromide (Himedia, India) using a DNA marker of $100 \mathrm{bp}$ (Himedia; India). The gels were visualized, photographed and documented in UV illuminator (Bio-Rad; Germany).

\section{Plasmid Profiling}

Isolates were examined for their plasmid content as previously described by [44] with slight modification. Briefly, plasmid DNAs were extracted from E. coli isolates by alkaline lysis method [3]. Briefly, $1.5 \mathrm{ml}$ of peptone water broth culture of single bacterial colony grown overnight at $37^{\circ} \mathrm{C}$ were centrifuged at room temperature. Collected cell pellets were then resuspended with glucose tris EDTA solution and placed on ice after adding $\mathrm{NaOH} / \mathrm{SDS}$ solution and sodium acetate solution consecutively. The preparations were then centrifuged at 13000rpm for 3 minutes and the supernatant was precipitated with ethanol to get the plasmid DNA. This plasmid DNA was washed and resuspended in TE buffer. Plasmid extracts were then separated by gel electrophoresis on $0.7 \%$ agarose gel alongside $1 \mathrm{kbp}$ marker in $1 \mathrm{X}$ TAE buffer at 10 volts per $\mathrm{cm}$ for 3 hours. The gels were then stained with ethidium bromide, visualized and documented by Geldocumentation system (BioRad; Germany).

\section{Statistical Methods}

Statistical analysis was made to generate descriptive statistics and look for associations among the different variables. The statistical evaluation was done using the SPSS for Windows package version 21.5.1 (SPSS Inc., Chicago, USA). Initially, the occurrence of each virulence factor and plasmids was described using descriptive statistics based on their frequency and average distribution. Moreover, any 
potential association between plasmid profile, resistance phenotype and virulence phenotype were assessed by applying multivariable logistic regression, as previously applied by Johnson et al. [21] and Chandran and Mazumder [10]. Levels of association between respective variables were considered significant at $p<0.05$. Finally, principal coordinates analysis $(P C o A)$, a multivariate technique related to correspondence analysis that enables plotting the major patterns within a dataset, was used to visualize the level of similarity of isolates based on all variables (consisting; each of virulence factors, plasmids and antibiotic resistance traits) and the values for pairwise combinations of the first two axes were plotted as a series of Cartesian grids, to show the relationship among the variables in two-dimensional space according to Johnson et al. [21]. Multivariate analysis of variance (MANOVA) was also conducted to determine whether the groups differed significantly according to the three PCoA axes as previously applied by Johnson et al. [21].

\section{Declarations}

\section{Acknowledgements}

We would like to thank the Institute of Biotechnology, Addis Ababa University; and College of Natural \& Computational Sciences, Addis Ababa University, for providing required working spaces for the different aspects of this work.

\section{Authors' Contributions}

TST was responsible for the study design, financing the research work, supervising the laboratory work and data analysis with a major contribution in writing the manuscript; AE performed all of the laboratory work and interpretation of the data in this work; $\mathrm{YU}$ was involved in the collection and partial analysis of samples to implement the study. All authors read and approved the final manuscript.

\section{Funding}

This work was financially supported by the thematic research project "Pneumonia, diarrhea and mastitis in food animals", Addis Ababa University, led by Tesfaye Sisay Tessema.

\section{Availability of data and materials}

The datasets used during the current study can be obtained from the corresponding author on request.

\section{Ethics approval and consent to participate}

This study was conducted on stored $E$. coli isolates. In addition, the research proposal was publicly defended and conduct of the experiment was permitted by the Graduate Committee of the Institute of Biotechnology, Addis Ababa University, Addis Ababa, Ethiopia.

\section{Competing interests}


Not applicable.

\section{Authors details}

${ }^{1}$ College of Natural \& Computational Sciences, Addis Ababa University, P.O.Box $=1176$, Addis Ababa, Ethiopia; E-mail address: abinetendale@gmail.com; ${ }^{2}$ College of Agriculture, Woldia University, Woldia, Ethiopia; E-mail address:yimermktr21@gmail.com; ${ }^{3}$ Institute of Biotechnology, Addis Ababa University, P.O.Box=1176, Addis Ababa, Ethiopia; E-mail address: tesfu74@yahoo.com

\section{References}

The references were omitted in this version of the paper.

\section{Tables}

Table 1 List of primers used for screening virulence genes in E. coli isolates.

\begin{tabular}{|c|c|c|c|c|}
\hline Primer code & Primer sequence 5'- $3^{\prime}$ & Target gene & \multicolumn{2}{|c|}{ Product size (bp) Reference } \\
\hline EAE-f & AAACAGGTGAAACTGTTGCC & $e a e A$ & 454 & Khan et al. (2002) \\
\hline EAE-r & СTCTGCAGATTAACCTCTGC & \multirow{3}{*}{ stx 1} & & \\
\hline EVC-f & ATCAGTCGTCACTCACTGGT & & 349 & Pal et al. (1999) \\
\hline EVC-r & CTGCTGTCACAGTGACAAA & & & \\
\hline EVT-f & CAACACTGGATGATCTCAG & \multirow[t]{2}{*}{ stx2 } & 112 & Pal et al. (1999) \\
\hline EVT-r & СССССТСААСТGСТААТА & & & \\
\hline BFPF & AATGGTGCTTGCGCTTGCTGC & $b f p A$ & 324 & Gunzburg et al. (1995) \\
\hline BFPR & GCCGCTTTATCCAACCTGGTA & & & \\
\hline EAEC F & CTGGCGAAAGACTGTATCAT & aatA & 630 & Schmidt et al.(1995) \\
\hline EAEC R & CAATGTATAGAAATCCGCTGTT & & & \\
\hline EHEC-F & ACGATGTGGTTTATTCTGGA & hlyA & 167 & \\
\hline EHEC-R & CTTCACGTCACCATACATAT & & & \\
\hline
\end{tabular}

Key: eaeA, E. coli attaching effacing gene; stx1, Shiga-like toxin I gene; stx2, Shiga-like toxin II gene; ehlyA, E. colihemolysin gene; bfpA, bundle forming pili gene. 
Table 2 Distribution of virulence genes in E. coli isolated from diarrheic calves $(n=73)$

\begin{tabular}{|c|c|c|}
\hline \multirow[t]{2}{*}{ Virulence factor } & \multicolumn{2}{|l|}{ Samples } \\
\hline & № of positive isolates (n) & Frequency (\%) \\
\hline$e a e A$ & 43 & 58.9 \\
\hline stx 1 & 25 & 34.25 \\
\hline stx 2 & 18 & 24.66 \\
\hline ehlyA & 17 & 23.29 \\
\hline$b f p A$ & 9 & 12.33 \\
\hline aatA & 4 & 5.48 \\
\hline
\end{tabular}

Key: eaeA, E. coli attaching effacing gene; stx1, Shiga-like toxin I gene; stx2, Shiga-like toxin II gene; ehlyA, E. colihemolysin gene; bfpA, bundle forming pili gene.

Table 3 Virulence profiles identified based on combination of virulence genes in $E$. coli isolates. 


\begin{tabular}{|c|c|c|c|c|c|c|c|c|}
\hline \multirow[t]{2}{*}{ No. of Isolates } & \multirow[t]{2}{*}{ Proportion (\%) } & \multicolumn{6}{|c|}{ Virulence genes } & \multirow[t]{2}{*}{ Virulence profile (VP) } \\
\hline & & eaeA & stx2 & stx 1 & $b f p A$ & ehlyA & aatA & \\
\hline 2 & 3.92 & & & & & & + & I \\
\hline 1 & 1.96 & & & + & & & & II \\
\hline 12 & 23.53 & + & & & & & & III \\
\hline 2 & 3.92 & & & + & & & + & IV \\
\hline 3 & 5.88 & & + & + & & & & $\mathrm{V}$ \\
\hline 9 & 17.65 & + & & & + & & & VI \\
\hline 2 & 3.92 & + & & + & & & & VII \\
\hline 3 & 5.88 & + & + & & & + & & VIII \\
\hline 5 & 9.80 & + & & + & & + & & IX \\
\hline 3 & 5.88 & + & + & + & & & & $\mathrm{X}$ \\
\hline 9 & 17.65 & + & + & + & & + & & XI \\
\hline
\end{tabular}

Key: eaeA, E. coli attaching effacing gene; stx1, Shiga-like toxin I gene; stx2, Shiga-like toxin II gene; ehlyA, E. colihemolysin gene; bfpA, bundle forming pili gene.

Table 4 Plasmid distribution frequency in $E$. coli isolates $(\mathrm{N}=30)$ 


\begin{tabular}{ccc}
\hline Plasmid Size \pm SD (Kbp) & Incidence & $\%$ frequency \\
\hline $2.6 \pm 0.14$ & 3 & 10 \\
$3.1 \pm 0.26$ & 10 & 33 \\
\hline $5.0 \pm 0.31$ & 5 & 17 \\
\hline $7.8 \pm 0.59$ & 10 & 33 \\
$11.8 \pm 0.82$ & 7 & 23 \\
\hline $14.3 \pm 1.1$ & 7 & 23 \\
\hline $16.2 \pm 1.4$ & 14 & 47 \\
\hline $34.6 \pm 1.7$ & 30 & 20 \\
\hline $42.5 \pm 2.1$ & 6 & 700 \\
\hline 4.17 & & \\
\hline
\end{tabular}

Table 5 Distribution of plasmids in $E$. coli isolates among the three sample sites.

\begin{tabular}{|c|c|c|c|c|c|c|c|c|c|c|c|}
\hline \multirow[t]{2}{*}{ Sample Area (N) } & \multicolumn{11}{|c|}{ Plasmid size (Kbp) } \\
\hline & 2.5 & 3.1 & 5.0 & 7.8 & 11 & 14.3 & 16.2 & 34.6 & 42.5 & 98.2 & req. $(\mathrm{N})$ \\
\hline MT (11) & 1 & 3 & 1 & 2 & 0 & 4 & 2 & 0 & 3 & 11 & 8 \\
\hline$F(9)$ & 0 & 1 & 1 & 2 & 2 & 0 & 2 & 1 & 0 & 9 & 16 \\
\hline DT (10) & 1 & 4 & 0 & 4 & 0 & 1 & 6 & 0 & 2 & 10 & 36 \\
\hline
\end{tabular}

Key. MT: Muketuri, F: Fiche, DT: Debretsige

Table 6 Plasmid distribution profile in $E$. coli isolates $(\mathrm{N}=30)$. 


\begin{tabular}{|c|c|c|c|}
\hline No. of Plasmid & Plasmid profile & $\ddot{\mathbf{x}}$ Plasmid Size (Kbp) & Number of Isolates \\
\hline 1 & I & 98.2 & 13 \\
\hline \multirow[t]{3}{*}{2} & II & $98.2,11.8$ & 1 \\
\hline & III & $98.2,16.2$ & 1 \\
\hline & IV & $98.2,16.2,7.8$ & 3 \\
\hline \multirow[t]{3}{*}{3} & $\mathrm{~V}$ & $98.2,14.3,3.1$ & 1 \\
\hline & VI & $98.2,16.2,3.1$ & 2 \\
\hline & VII & $98.2,42.5,14.3,2.5$ & 2 \\
\hline \multirow[t]{4}{*}{4} & VIII & $98.2,16.2,7.8,3.1$ & 3 \\
\hline & IX & $98.2,42.5,14.3,5$ & 1 \\
\hline & $\mathrm{X}$ & $98.2,34.6,11.8,5$ & 1 \\
\hline & $\mathrm{XI}$ & $98.2,42.5,14.3,7.8,3.1$ & 1 \\
\hline 5 & XII & $98.2,42.5,16.2,7.8,3.1$ & 1 \\
\hline
\end{tabular}

Key. ẍ Plasmid Size, mean plasmid size

\section{Figures}



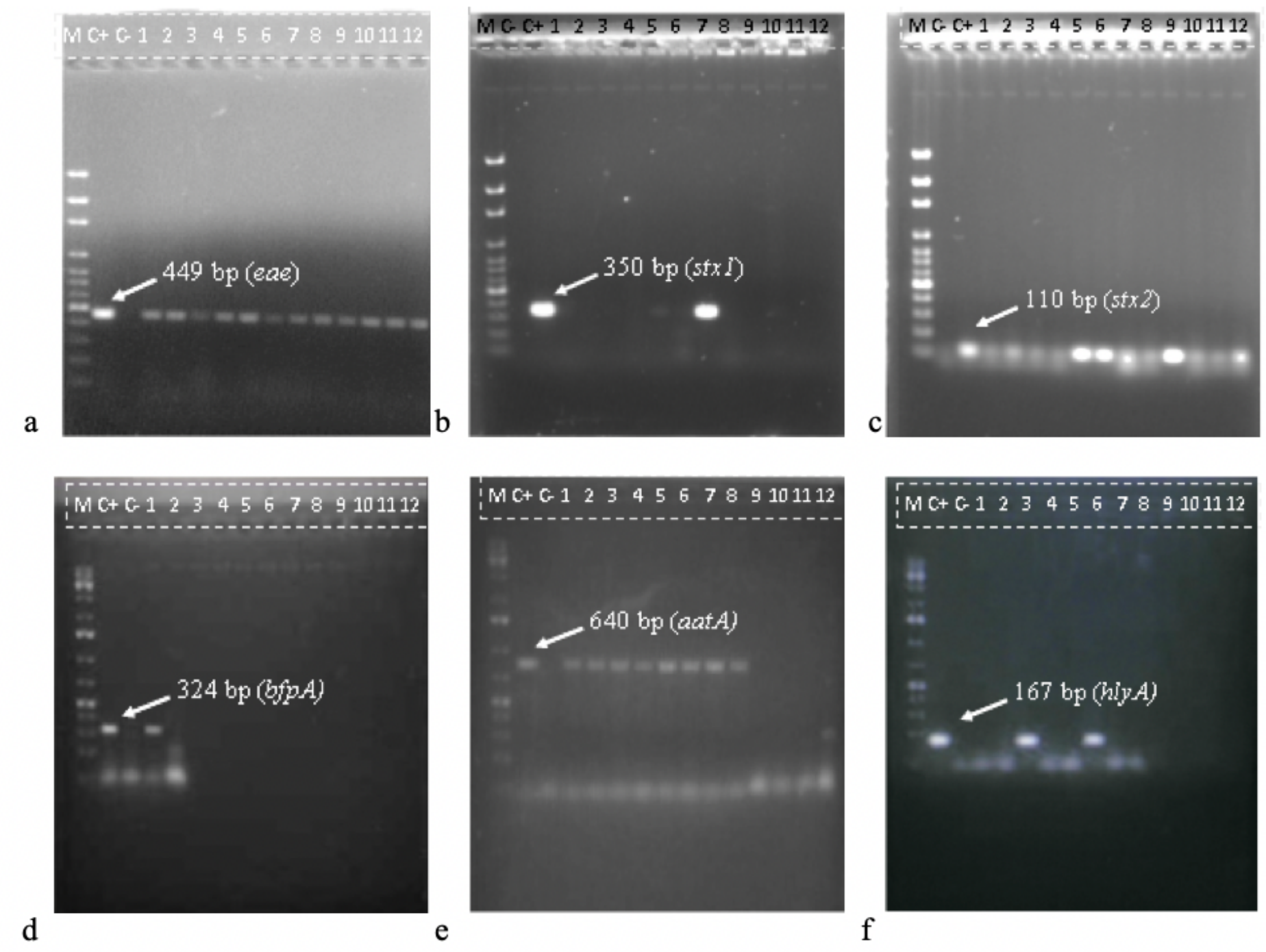

\section{Figure 1}

Representative gels for PCR product of E. coli virulence genes. a) eaeA (449 bp), b) stx1 (350 bp), c) stx2 (110 bp), d) bfpA (324), e) aatA (640) and f) hlyA (167). Lane M; DNA size marker (100 bp), lane; 1-12 $\mathrm{PCR}$ product of isolates, lane $\mathrm{C}+$ positive control and $\mathrm{C}-$ negative control. The relative position of each PCR products is indicated by arrow heads. 


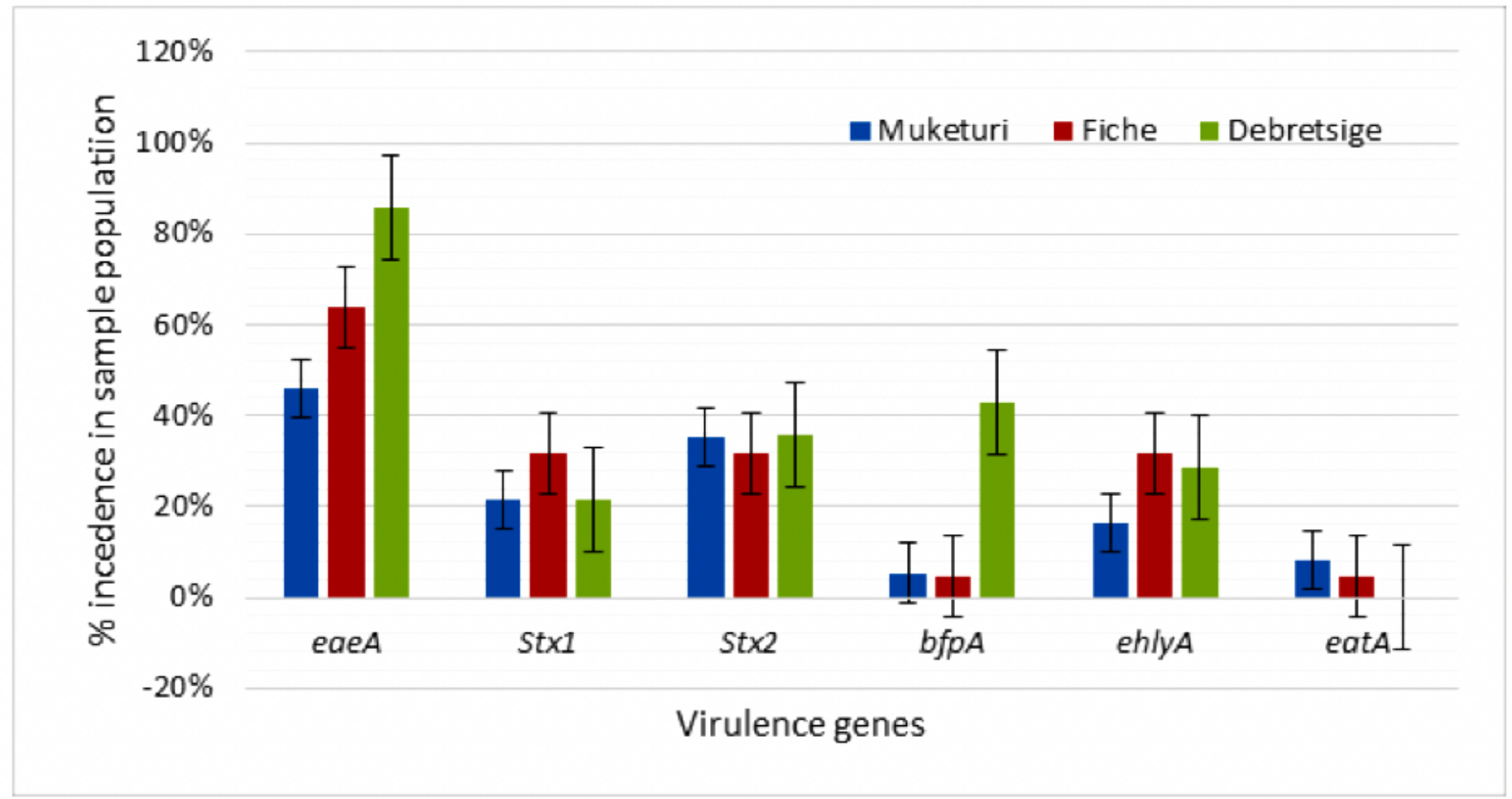

Figure 2

Spatial distribution of E. coli virulence genes among the three sample sites. Key: eaeA, E. coli attaching effacing gene; stx1, Shiga-like toxin I gene; stx2, Shiga-like toxin II gene; ehlyA, E. coli hemolysin gene; bfpA, bundle forming pili gene. 


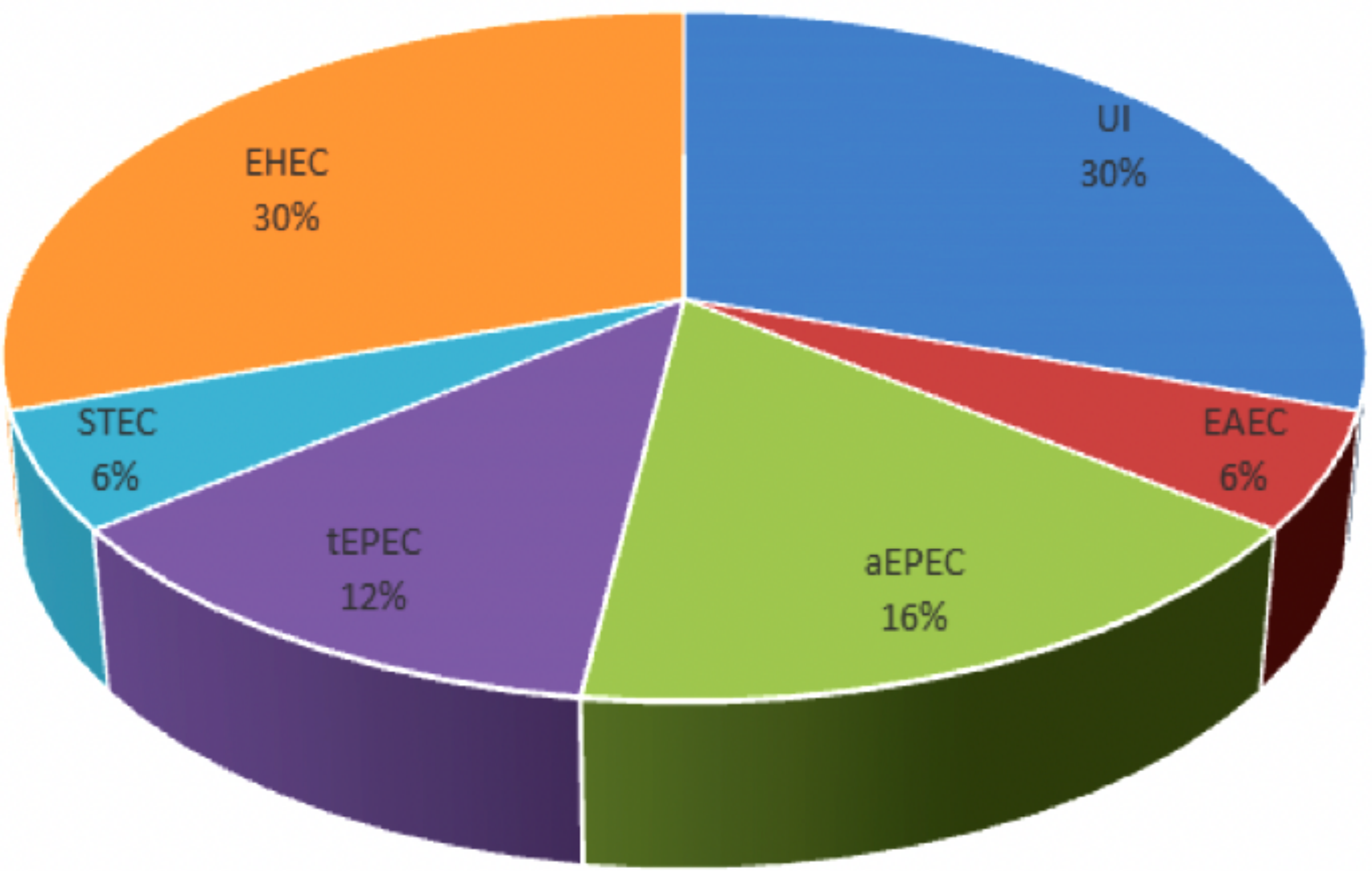

Figure 3

Frequency of different diarrheagenic E. coli pathotypes in sample isolates Key: EAEC, Enteroaggregative E. coli; EHEC, Enterohemorrhagic E. coli; aEPEC, Atypical enteropathogenic E. coli; tEPEC, typical enteropathogenic E. coli; STEC, Shiga-like toxin producing E. coli; UI, unidentified E. coli 


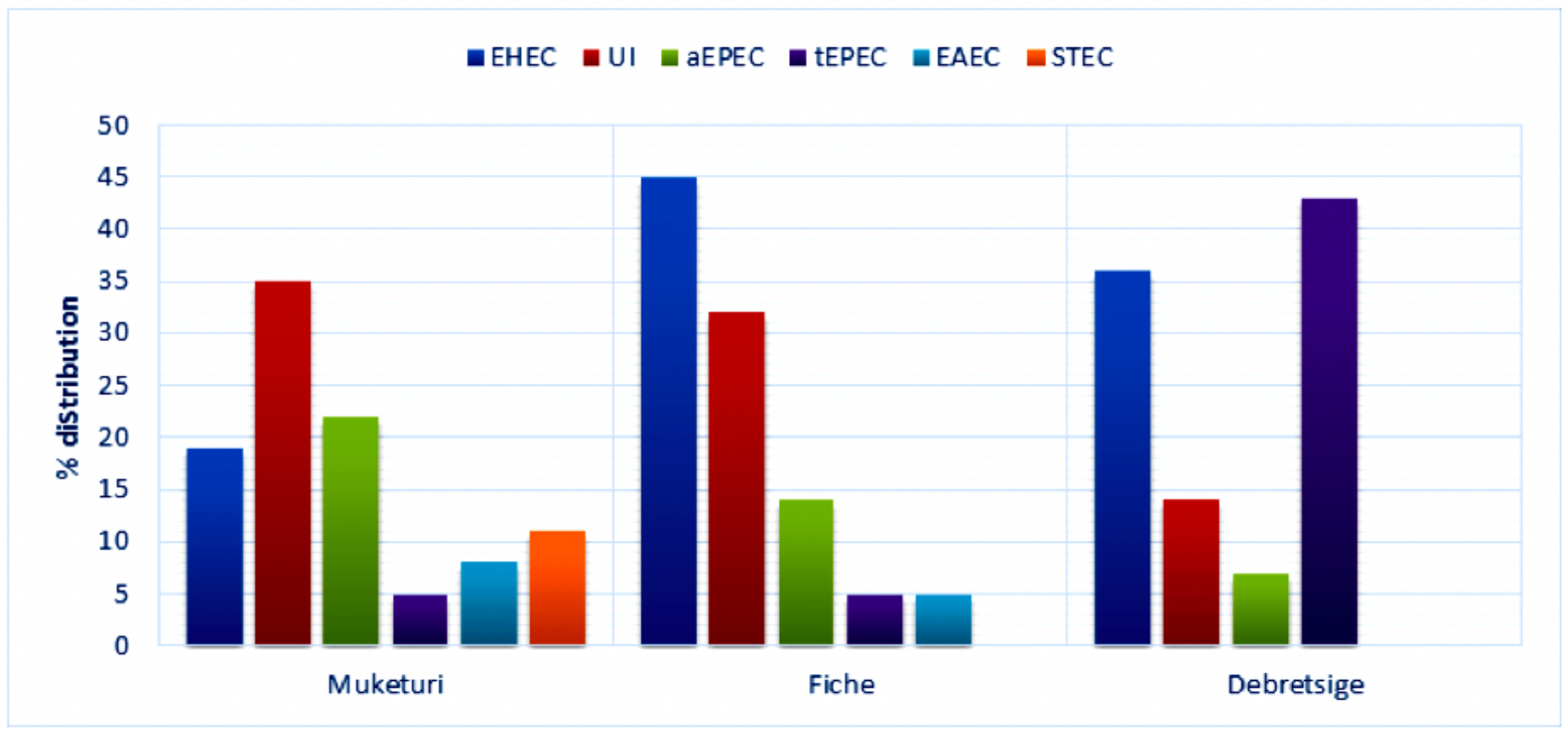

Figure 4

Distribution of E. coli pathotypes among the three sampling sites. 


\section{$\begin{array}{lllllllllllllll}M & 1 & 2 & 3 & 4 & 5 & 6 & 7 & 8 & 9 & 10 & 11 & 12 & 13 & 14\end{array}$}

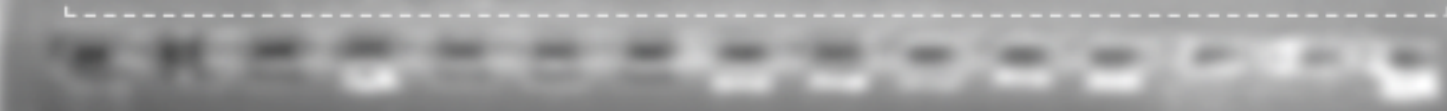

\section{0} 8000

3000
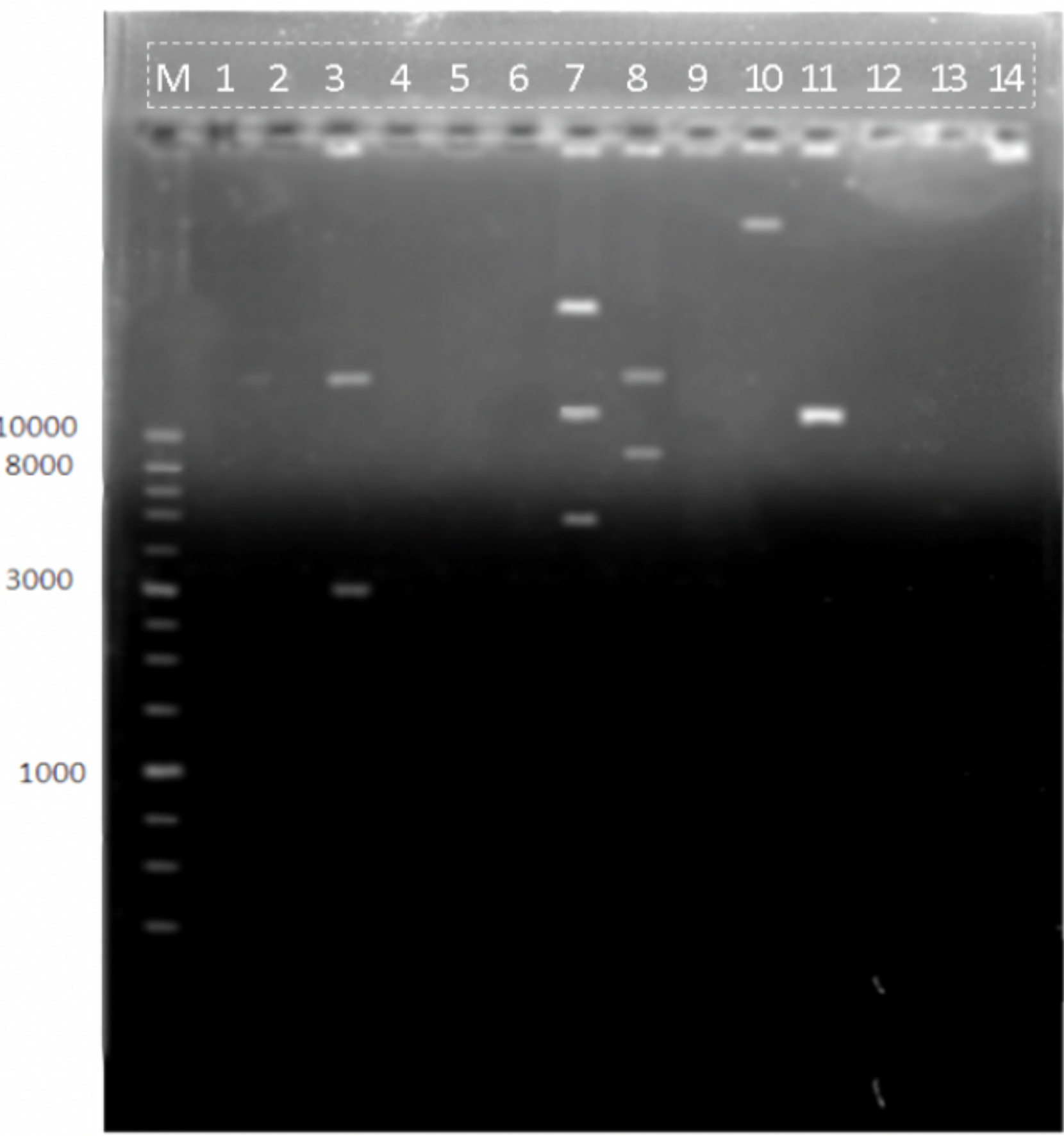

\section{Figure 5}

Representative plasmids following electrophoresis on $0.7 \%$ agarose gel. Lane M: DNA size marker (1 $\mathrm{Kbp})$, lane 1-14: isolated plasmids. 


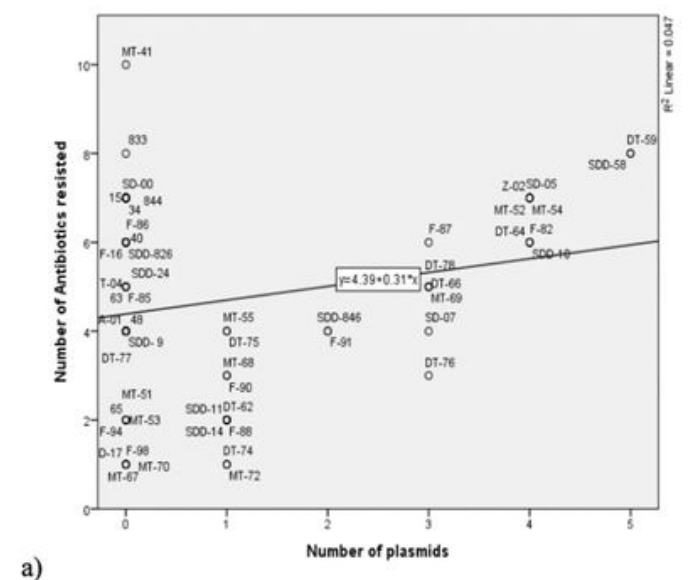

a)

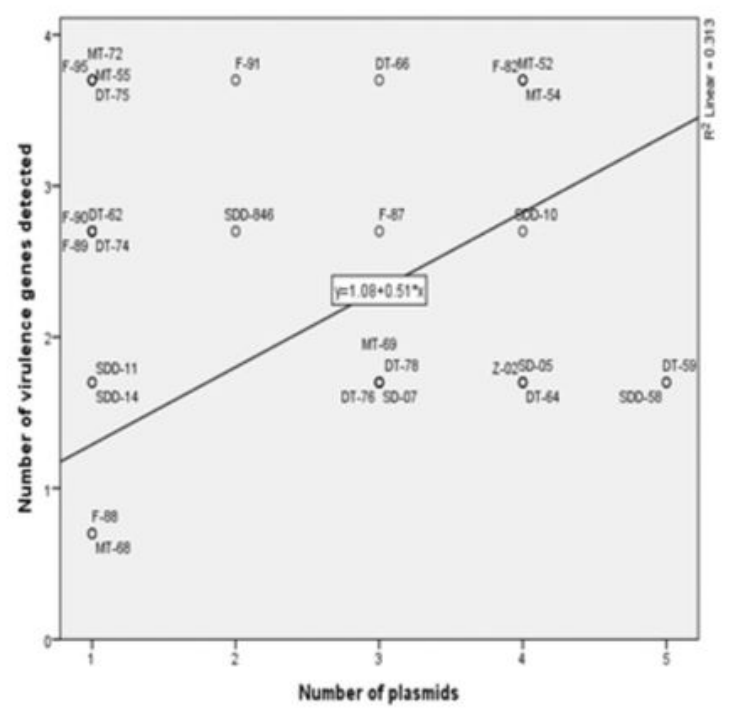

b)

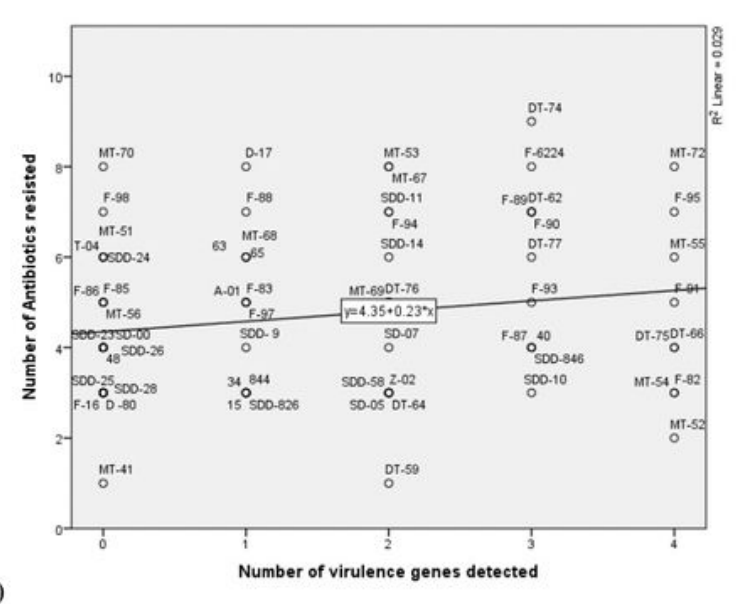

\section{Figure 6}

Scatter Plot showing the relationship between plasmids, virulence gene and antibiotic resistance. a) number of plasmids vs number of virulence factor gens detected $b$ ) number of plasmids vs level of antimicrobial resistance c) number of virulence factor gens detected vs level of antimicrobial resistance. 


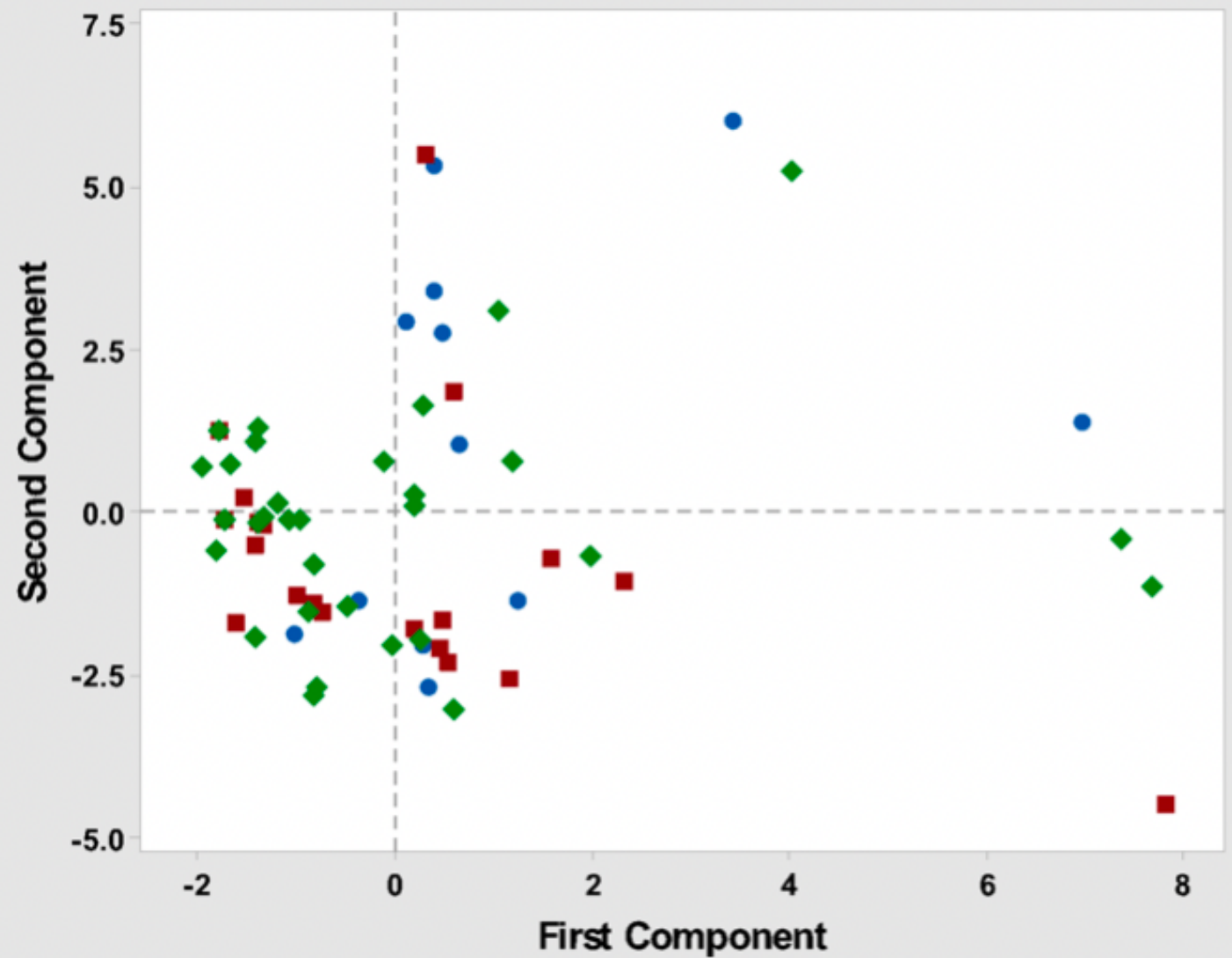

First Component

Figure 7

Score plot showing the first two components based on PCoA of isolates based on distribution of plasmids, virulence gene and antibiotic resistance traits. 